Supporting information for:

\title{
Particles Emissions from Laser Printers: have they decreased?
}

Lidia Morawska ${ }^{\mathrm{a},}$ *, Meng Xiu ${ }^{\mathrm{a}}$, Congrong He ${ }^{\mathrm{a}}$, Giorgio Buonanno ${ }^{\mathrm{a}}$,, Peter McGarry Bastien Maumy ${ }^{\mathrm{c}}$, Luca Stabile ${ }^{\mathrm{b}}$, Phong K Thai ${ }^{\mathrm{a}}$

a International Laboratory for Air Quality and Health, Queensland University of Technology, Brisbane, Qld 4001, Australia

${ }^{\mathrm{b}}$ Department of Civil and Mechanical Engineering, University of Cassino and Southern Lazio, via G. di Biasio 43, 03043 Cassino, FR, Italy

${ }^{c}$ EDF Energy R\&D UK Centre Ltd, 80 Victoria Street, London, SW1E 5JL, UK

${ }^{*}$ Corresponding author:

Lidia Morawska, email: 1.morawska@qut.edu.au 

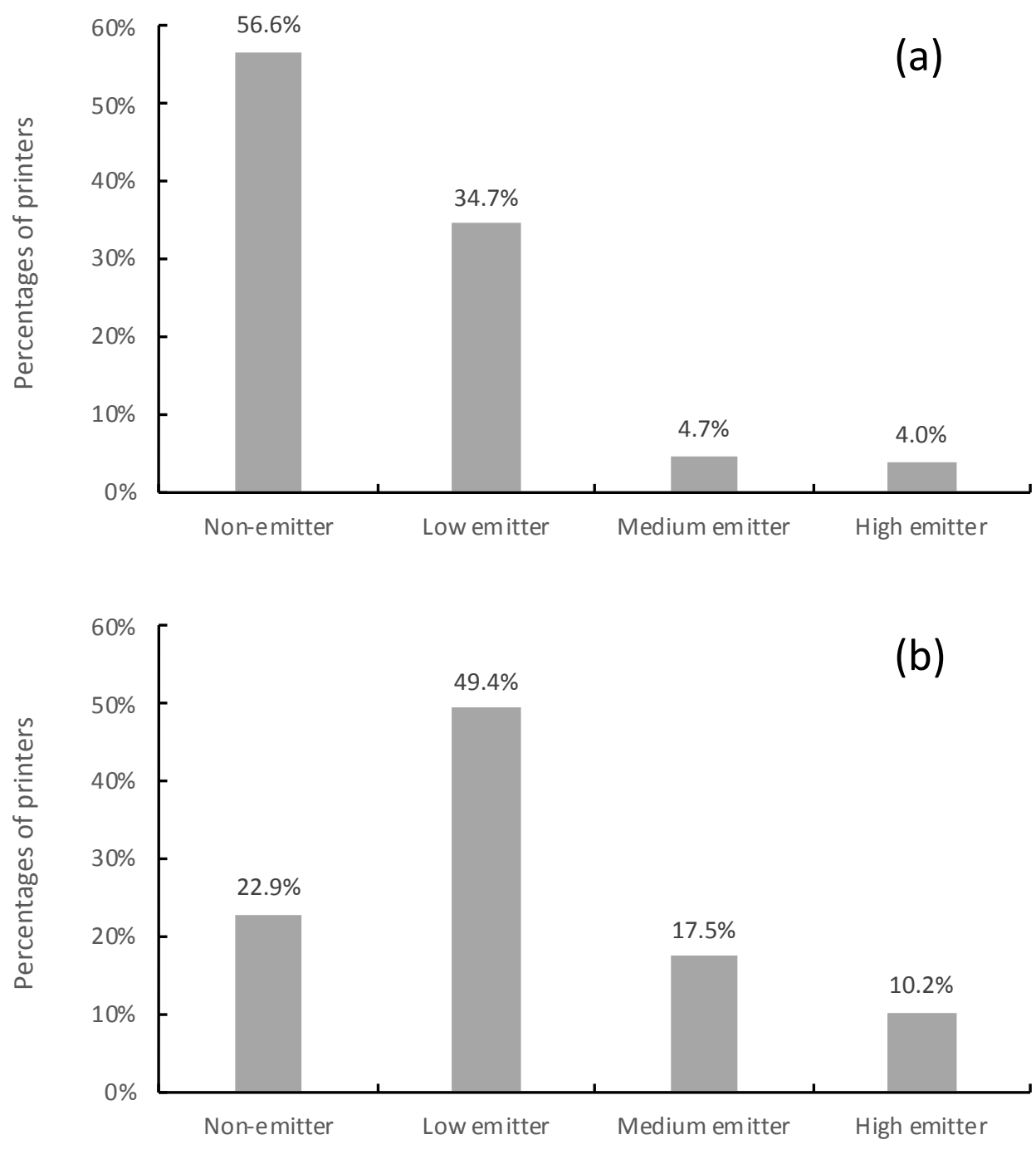

Figure S1. Summary of results for printer emission investigations: (a) based on the ratio of PNC peak values to background values in Brisbane and Cassino, and (b) based on the ratio of VOC peak values to background values in Brisbane 

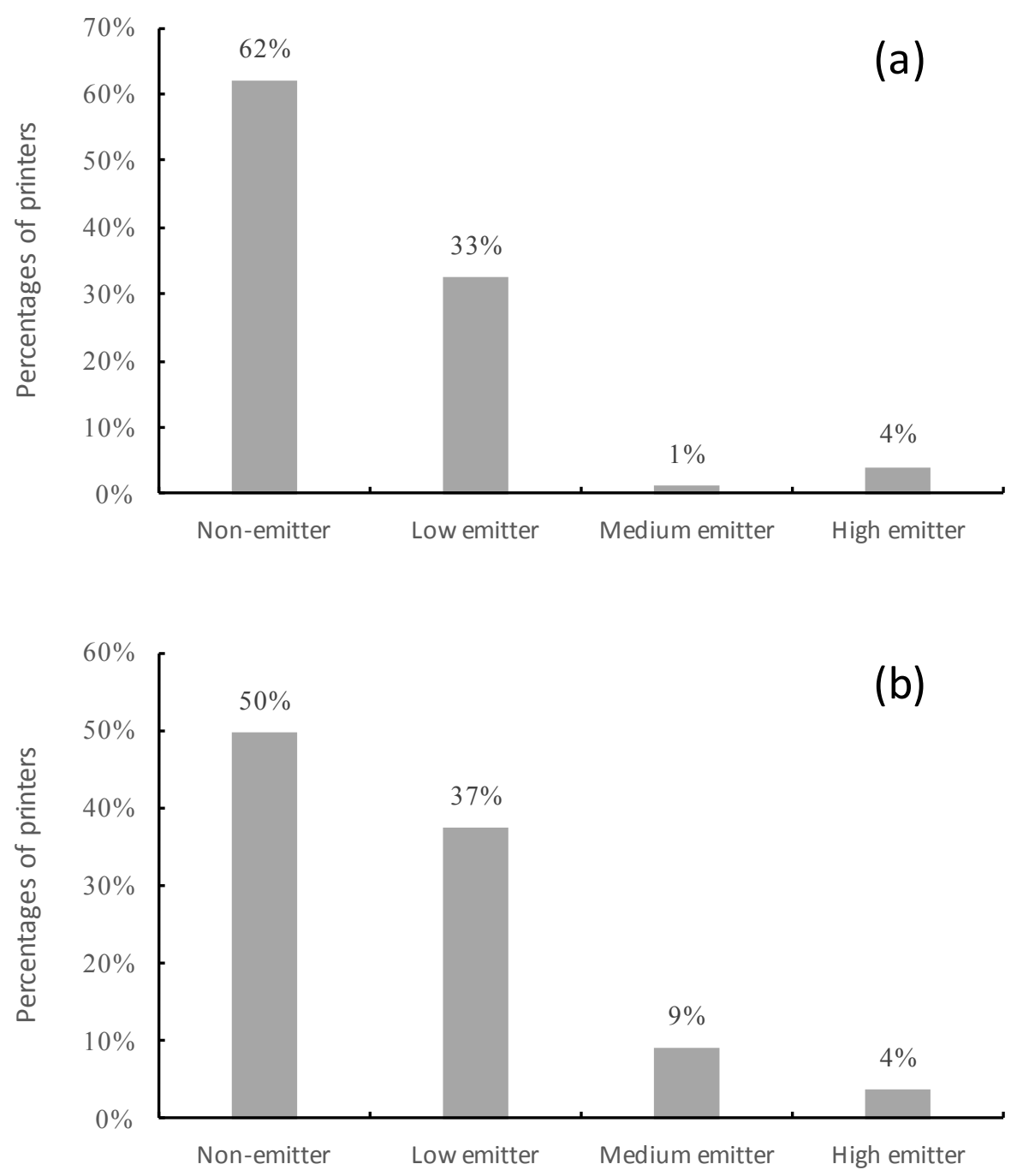

Figure S2. Summary of results for printer emission investigations, based on the ratio of PNC peak values to background values in Brisbane (a) and Cassino (b). 
Table S1. Summary of results for printer emission investigations in Brisbane and Cassino, based on the ratio of particle number concentration peak value emitted by the printer to the background value.

\begin{tabular}{|c|c|c|c|}
\hline $\begin{array}{l}\text { Non-emitter } \\
(\text { ratio } \leq 1.1)\end{array}$ & $\begin{array}{l}\text { Low emitter } \\
(1.1<\text { ratio } \leq 5)\end{array}$ & $\begin{array}{l}\text { Medium emitter } \\
(5<\text { ratio } \leq 10)\end{array}$ & $\begin{array}{l}\text { High emitter } \\
(\text { ratio }>10)\end{array}$ \\
\hline ApeosPort-IV B3070 (1) & ApeosPort-IV 4070 (1) & ApeosPort-IV C3375 & Dell 2130 cn (1) \\
\hline ApeosPort-IV B4070 (1) & $\begin{array}{l}\text { ApeosPort-IV B4070 } \\
\text { (1) }\end{array}$ & $\begin{array}{l}\text { Brother HL-1212W } \\
\text { (1) }\end{array}$ & DocuPrint C2200 (2) \\
\hline ApeosPort-IV C3370 (6) & $\begin{array}{l}\text { ApeosPort-IV C3370 } \\
\text { (5) } \\
\text { ApeosPort-IV C } 3375\end{array}$ & $\begin{array}{l}\text { Canon LBP } 7210 \text { Cdn } \\
\text { (1) }\end{array}$ & $\begin{array}{l}\text { DocuPrint CM305df } \\
\text { (1) }\end{array}$ \\
\hline ApeosPort-IV C3375 (1) & $\begin{array}{l}\text { (1) } \\
\text { ApeosPort-IV C4430 }\end{array}$ & $\begin{array}{l}\text { Dell 1710n (1) } \\
\text { Epson Aculaser }\end{array}$ & $\begin{array}{l}\text { DocuPrint P355d (1) } \\
\text { Epson Aculaser C2900 }\end{array}$ \\
\hline ApeosPort-IV C4430 (1) & $\begin{array}{l}\text { (2) } \\
\text { ApeosPort-IV C4470 }\end{array}$ & $\begin{array}{l}\text { M2000D L521A (1) } \\
\text { Epson Aculaser }\end{array}$ & $\begin{array}{l}\text { (1) } \\
\text { Epson Aculaser }\end{array}$ \\
\hline ApeosPort-IV C4470 (22) & $\begin{array}{l}\text { (4) } \\
\text { ApeosPort-IV C4475 }\end{array}$ & M2000D L521B (1) & M2300DN L522B (1) \\
\hline ApeosPort-IV C4475 (20) & (10) & $\begin{array}{l}\text { HP Laserjet } 1320 \text { (1) } \\
\text { Hp LaserJet P2014 }\end{array}$ & $\begin{array}{l}\text { Phaser } 4600 \text { (2) } \\
\text { Ricoh Aficio MP }\end{array}$ \\
\hline ApeosPort-V C3375 (6) & ApeosPort-V C3375 (5) & $\begin{array}{l}\text { (1) } \\
\text { Hp LaserJet P3015 }\end{array}$ & $\begin{array}{l}\text { C2050 (1) } \\
\text { Samsung ML-2851ND }\end{array}$ \\
\hline ApeosPort-V C4475 (12) & ApeosPort-V C4475 (2) & $\begin{array}{l}\text { (1) } \\
\text { Samsung ML- }\end{array}$ & $\begin{array}{l}\text { (1) } \\
\text { Xerox Workcentre }\end{array}$ \\
\hline Brother MFC-8510DN (1) & Canon ir1600 (1) & $\begin{array}{l}\text { 2855ND (1) } \\
\text { Samsung Xpress }\end{array}$ & $6505(1)$ \\
\hline Brother MFC-9120CN (1) & Canon ir2018 (1) & M2070 (1) & \\
\hline $\begin{array}{l}\text { Brother MFC-L2700DW } \\
\text { (1) } \\
\text { Canon i-SENSYS } \\
\text { MF4270 (1) } \\
\text { Canon i-Sensys MF4340d } \\
\text { (1) }\end{array}$ & $\begin{array}{l}\text { Dell 1710n (1) } \\
\text { DocuPrint C5005d (3) } \\
\text { DocuPrint CM305df (1) }\end{array}$ & $\begin{array}{l}\text { Xerox Workcentre } \\
3210(1) \\
\text { Xerox Workcentre } \\
6505 D N(1) \\
\text { Xerox Workcentre } \\
6605 \text { (1) }\end{array}$ & \\
\hline DocuPrint C5005d (3) & Docuprint M355df (1) & & \\
\hline DocuPrint P355d (2) & DocuPrint P355d (1) & & \\
\hline Epson Aculaser CX17 (2) & $\begin{array}{l}\text { DocuPrint P455d (1) } \\
\text { Epson Aculaser }\end{array}$ & & \\
\hline Epson Aculaser CX37 (1) & $\mathrm{C} 2800 \mathrm{~N}(2)$ & & \\
\hline $\begin{array}{l}\text { Epson Aculaser M1200 } \\
\text { (1) }\end{array}$ & $\begin{array}{l}\text { Epson Aculaser } \\
\text { C2900N (1) } \\
\text { Epson Aculaser M1200 }\end{array}$ & & \\
\hline Epson AL-M200DN (2) & $\begin{array}{l}\text { L471A (1) } \\
\text { Epson Aculaser }\end{array}$ & & \\
\hline HP Laserjet 1150 (1) & $\begin{array}{l}\text { M2000D L521A (1) } \\
\text { Epson Aculaser }\end{array}$ & & \\
\hline Hp LaserJet CP1515n (1) & M200ND (1) & & \\
\hline $\begin{array}{l}\text { Hp LaserJet M1005 MFP } \\
\text { (1) }\end{array}$ & $\begin{array}{l}\text { Epson AL-M200DN } \\
\text { 77733A (1) }\end{array}$ & & \\
\hline Hp LaserJet M1120 MFP & HP color LaserJet & & \\
\hline $\begin{array}{l}\text { (2) } \\
\text { Hp LaserJet M1132 MFP }\end{array}$ & $\begin{array}{l}4700 \mathrm{dtn}(1) \\
\text { HP color laserjet }\end{array}$ & & \\
\hline $\begin{array}{l}\text { (3) } \\
\text { Hp LaserJet M1319 f }\end{array}$ & CP3525dn (1) & & \\
\hline MFP (1) & HP LaserJet 3055 (1) & & \\
\hline
\end{tabular}




\begin{tabular}{|c|c|}
\hline \multirow{3}{*}{ Hp LaserJet P1005 (1) } & Hp LaserJet CM2320 \\
\hline & fxi MFP (1) \\
\hline & HP LaserJet CP3525dn \\
\hline Hp LaserJet P1006 (2) & $(1)$ \\
\hline & Hp LaserJet M1522n \\
\hline Hp LaserJet P1102 (1) & $\begin{array}{l}\text { (1) } \\
\text { HP LaserJet }\end{array}$ \\
\hline HP LaserJet P1505n (1) & M3027xMFP (1) \\
\hline Hp LaserJet P1606dn (1) & Hp LaserJet P2014 (1) \\
\hline Hp LaserJet P2015 (1) & Hp LaserJet P2015 (2) \\
\hline HP Laserjet P2035 (1) & $\begin{array}{l}\text { Hp LaserJet P2055d (2) } \\
\text { Hp LaserJet P2055dn }\end{array}$ \\
\hline Hp LaserJet P2055dn (1) & $\begin{array}{l}\text { (2) } \\
\text { HP Laserjet Pro } 400\end{array}$ \\
\hline Hp LaserJet P4014 (1) & M401d (1) \\
\hline Hp LaserJet Pro 200 & Hp LaserJet Pro 400 \\
\hline Color M251nw (1) & M401d (1) \\
\hline Hp LaserJet Pro 400 & HP Laserjet Pro 400 \\
\hline M401a (1) & M401dne (1) \\
\hline Hp LaserJet Pro 400 & Hp LaserJet Pro 400 \\
\hline M401dne (2) & MFP M425dn (1) \\
\hline HP LaserJet Pro & Hp LaserJet Pro MFP \\
\hline CM1415fr Color MFP (1) & M476dw (1) \\
\hline Hp LaserJet Pro MFP & \\
\hline M125a (1) & HP M451 (1) \\
\hline Hp LaserJet Pro MFP & Konica Minolta bizhub \\
\hline M477fdw (1) & C364e (6) \\
\hline Hp LaserJet1320 (1) & $\begin{array}{l}\text { Konica Minolta DK- } \\
506 \text { (1) }\end{array}$ \\
\hline Hp LaserJet3050 (1) & Phaser 3435 DN (1) \\
\hline Hp LaserJet3055 (1) & Phaser 4620 (1) \\
\hline Konica Minolta bizhub & Ricoh Aficio MP \\
\hline C364e (14) & C2005 (2) \\
\hline Lexmark E260dn (2) & $\begin{array}{l}\text { Ricoh Aficio MP C305 } \\
\text { (1) } \\
\text { RICOH Aficio MP }\end{array}$ \\
\hline Lexmark MS811dn (1) & C45024 (1) \\
\hline Lexmark MX410de (1) & $\begin{array}{l}\text { Ricoh Aficio SP C250sf } \\
\text { (1) }\end{array}$ \\
\hline Lexmark X264nd (1) & $\begin{array}{l}\text { RICOH Pro 8120s (1) } \\
\text { Samsung CLP-365W }\end{array}$ \\
\hline Phaser 3435 (5) & $\begin{array}{l}\text { (1) } \\
\text { Samsung CLX-3305FW }\end{array}$ \\
\hline Phaser 4600 (2) & (1) \\
\hline Phaser 4620 (1) & Samsung ML-1630 (1) \\
\hline Phaser 4620 (1) & $\begin{array}{l}\text { Samsung ML-2165 (1) } \\
\text { Samsung SCX 5835FN }\end{array}$ \\
\hline Ricoh Aficio MP 2550 (1) & $\begin{array}{l}\text { (1) } \\
\text { Samsung SCX-3405F }\end{array}$ \\
\hline Samsung CLP-365W (1) & (1) \\
\hline Samsung CLP-620ND (1) & $\begin{array}{l}\text { Samsung SCX-3405FW } \\
\text { (2) }\end{array}$ \\
\hline Samsung CLX-3185 (1) & $\begin{array}{l}\text { Samsung SCX-4300 } \\
\text { (1) }\end{array}$ \\
\hline
\end{tabular}




\begin{tabular}{|c|c|}
\hline Samsung ML-2010R (1) & $\begin{array}{l}\text { Samsung SCX-4623 } \\
\text { SERIES (1) } \\
\text { Samsung SCX-4623F }\end{array}$ \\
\hline Samsung ML-3310ND (2) & $\begin{array}{l}\text { (2) } \\
\text { Samsung Xpress }\end{array}$ \\
\hline Samsung SCX-4200 (1) & $\begin{array}{l}\text { C410W (1) } \\
\text { Samsung Xpress }\end{array}$ \\
\hline Samsung SCX-4300 (1) & M2675F (1) \\
\hline Samsung SCX-4521F (2) & Xerox Phaser 6280 (1) \\
\hline $\begin{array}{l}\text { Samsung SCX-4623F (2) } \\
\text { Samsung SCX-5835FN } \\
\text { (2) } \\
\text { Samsung Xpress M2022 } \\
\text { (1) } \\
\text { Samsung Xpress } \\
\text { M2875FD (1) } \\
\text { Xerox Workcentre } 3225 \\
\text { (3) } \\
\text { Xerox Workcentre } 4250 \\
\text { (1) } \\
\text { Xerox Workcentre } 5675 \\
\text { (1) } \\
\text { Xerox Workcentre } 6027 \\
\text { (1) } \\
\text { Xerox Workcentre } 7245 \\
\text { (1) } \\
\text { Xerox Workcentre } 7755 \\
\text { (1) }\end{array}$ & $\begin{array}{l}\text { Xerox Phaser } 6500(1) \\
\text { Xerox Workcentre } 6015 \\
(1) \\
\text { Xerox Workcentre } \\
\text { 6505DN (3) }\end{array}$ \\
\hline
\end{tabular}


Table S2. Summary of results for printer emission investigations in Brisbane, based on the ratio of VOC concentration peak value emitted by the printer to the background value.

\begin{tabular}{|c|c|c|c|}
\hline $\begin{array}{l}\text { Non-emitter } \\
(\text { ratio } \leq 1.1)\end{array}$ & $\begin{array}{l}\text { Low emitter } \\
(1.1<\text { ratio } \leq 5)\end{array}$ & $\begin{array}{l}\text { Medium emitter } \\
(5<\text { ratio } \leq 10)\end{array}$ & $\begin{array}{l}\text { High emitter } \\
(\text { ratio }>10)\end{array}$ \\
\hline $\begin{array}{l}\text { ApeosPort-IV B4070 } \\
\text { (1) } \\
\text { ApeosPort-IV C3370 } \\
\text { (3) } \\
\text { ApeosPort-IV C3375 } \\
\text { (1) } \\
\text { ApeosPort-IV C4470 } \\
\text { (4) } \\
\text { ApeosPort-IV C4475 } \\
\text { (7) } \\
\text { ApeosPort-V C3375 } \\
\text { (7) } \\
\text { ApeosPort-V C4475 } \\
\text { (4) } \\
\text { DocuPrint C5005d (1) } \\
\text { DocuPrint P355d (1) } \\
\text { DocuPrint P455d (1) } \\
\text { HP laser Jet } \\
\text { CP3525dn (1) } \\
\text { HP laser Jet P1505n } \\
\text { (1) } \\
\text { Konica Minolta } \\
\text { bizhub C364e (3) } \\
\text { Phaser 3435 DN (1) } \\
\text { Phaser 4600 (1) } \\
\text { RICOH Pro 8120s (1) }\end{array}$ & $\begin{array}{l}\text { ApeosPort-IV } 4070 \text { (1) } \\
\text { ApeosPort-IV B3070 (1) } \\
\text { ApeosPort-IV B4070 (1) } \\
\text { ApeosPort-IV C3370 (5) } \\
\text { ApeosPort-IV C3375 (2) } \\
\text { ApeosPort-IV C4430 (3) } \\
\text { ApeosPort-IV C4470 (11) } \\
\text { ApeosPort-IV C4475 (15) } \\
\text { ApeosPort-V C3375 (4) } \\
\text { ApeosPort-V C4475 (6) } \\
\text { Dell 2130 cn (1) } \\
\text { DocuPrint C5005d (3) } \\
\text { DocuPrint CM305df (2) } \\
\text { Docuprint M355df (1) } \\
\text { DocuPrint P355d (2) } \\
\text { HP color laser jet 4700dtn } \\
\text { (1) } \\
\text { HP Laser Jet 3055 (1) } \\
\text { HP Laser Jet M3027xMFP } \\
\text { (1) HP Laser Jet Pro CM1415fr } \\
\text { Color MFP (1) } \\
\text { Konica Minolta bizhub } \\
\text { C364e (12) } \\
\text { Konica Minolta DK-506 (1) } \\
\text { LexMark E260dn (1) } \\
\text { Phaser 3435 (2) } \\
\text { Phaser 4600 (2) } \\
\text { Phaser 4620 (1) } \\
\text { Phaser 4620 (1) }\end{array}$ & $\begin{array}{l}\text { ApeosPort-IV C3370 } \\
\text { (3) } \\
\text { ApeosPort-IV C4470 } \\
\text { (7) } \\
\text { ApeosPort-IV C4475 } \\
\text { (8) } \\
\text { Dell 1710n (1) } \\
\text { DocuPrint C2200 (1) } \\
\text { DocuPrint C5005d (1) } \\
\text { DocuPrint P355d (1) } \\
\text { Konica Minolta } \\
\text { bizhub C364e (2) } \\
\text { Phaser 3435 (2) } \\
\text { Phaser 4600 (1) } \\
\text { RICOH Aficio MP } \\
\text { C45024 (1) } \\
\text { WorkCentre 4250 (1) }\end{array}$ & $\begin{array}{l}\text { ApeosPort-IV C4470 } \\
\text { (4) } \\
\text { ApeosPort-IV C4475 } \\
\text { (3) } \\
\text { ApeosPort-V C4475 } \\
\text { (1) } \\
\text { Dell 1710n (1) } \\
\text { DocuPrint C2200 (1) } \\
\text { DocuPrint C5005d (1) } \\
\text { Konica Minolta } \\
\text { bizhub C364e (3) } \\
\text { Lexmark E260dn (1) } \\
\text { Phaser 3435 (1) } \\
\text { Phaser 4620 (1) }\end{array}$ \\
\hline
\end{tabular}

